\title{
Gl uso medicinal de cannabinoides no demostró un daro beneficio mientras que sí hubo asociación con un riesgo aumentado de presentar efectos adversos
}

Medicinal use of cannabinoids did not prove clear benefit while there was some association with increased risk of adverse events

\section{Objetivos}

Evaluar la evidencia disponible sobre los beneficios y efectos adversos (EA) relacionados al uso de cannabinoides para una amplia gama de indicaciones médicas.

\section{Diseño y fuente de datos}

Revisión sistemática y metaanálisis. Fueron examinadas 28 bases de datos y fuentes de literatura gris.

\section{Selección de estudios}

Se seleccionaron ensayos clínicos que hayan comparado el uso de cannabinoides contra tratamiento habitual, placebo o ningún
Whiting P y col. JAMA. 2015;313(24):2456-73. tratamiento para las siguientes indicaciones: náuseas y vómitos inducidos por quimioterapia, estimulación del apetito en pacientes con VIH/SIDA, dolor crónico neuropático o inducido por cáncer, espasticidad en esclerosis múltiple (EM) o paraplejia, depresión, trastornos de ansiedad, trastornos del sueño, psicosis, glaucoma y síndrome de Tourette.

No hubo restricción relacionada a la fecha de publicación o por idioma.

\section{Resultados Principales}

Se resumen en las tablas 1 y 2

Tabla 1. Resumen de los principales resultados de los metaanálisis para las principales indicaciones. Se agrega la valoración según el sistema GRADE sobre la calidad de evidencia de los mismos.

\begin{tabular}{l|c|c|c|c}
\multicolumn{1}{c|}{ Indicación / Resultado } & $\begin{array}{c}\text { No de estudios } \\
\mathbf{n}^{\circ} \text { de pacientes) }\end{array}$ & $\begin{array}{c}\text { Resultado } \\
\text { OR (IC95\%) }\end{array}$ & $\begin{array}{c}\text { A favor de } \\
\text { Calidad de evidencia } \\
\text { según GRADE }\end{array}$ & Cannabinoide \\
\hline $\begin{array}{l}\text { Supresión de náuseas y vómitos inducidos } \\
\text { por quimioterapia }\end{array}$ & $3(102)$ & $3,82(1,55 \mathrm{a} 9,42)$ & Cannabinoide & Moderada \\
\hline $\begin{array}{l}\text { Ganancia de } \geq \mathbf{2} \text { kg en seis semanas en } \\
\text { pacientes con VIH/SIDA }\end{array}$ & $1(88)$ & $2,2(0,68 \mathrm{a} 7,27)$ & Cannabinoide & Moderada \\
\hline $\begin{array}{l}\text { Reducción del dolor en un } \mathbf{3 0} \% \text { en pacientes } \\
\text { con dolor crónico }\end{array}$ & $8(1.370)$ & $1,41(0,99 \mathrm{a} 2,00)$ & Cannabinoide & Baja \\
\hline $\begin{array}{l}\text { Reducción de la espasticidad en un } \mathbf{5 0} \% \text { en } \\
\text { pacientes con EM o paraplejía }\end{array}$ & $2(519)$ & $1,40(0,81 \mathrm{a} 2,41)$ & & \\
\hline
\end{tabular}

OR: Odds ratio. IC95\%: intervalo de confianza del 95\%. GRADE: escala para valoración de la calidad de evidencia para la medida de resultado del conjunto de estudios incluidos. EM: esclerosis múltiple.

Tabla 2. . Probabilidad de presentar efectos adversos con el uso de cannabinoides versus placebo o fármaco activo.

\begin{tabular}{l|c|c}
\multicolumn{1}{c|}{ Categoría de EA } & $\mathbf{N}^{\circ}$ de estudios ( $\mathbf{n}^{\circ}$ de pacientes) & Resultado $\mathbf{~ O R}$ (IC95\%) \\
\hline Categoría general de efecto adverso & & $3,03(2,42 \mathrm{a} 3,80)$ \\
\hline -Cualquiera & $29(3.714)$ & $1,41(1,04 \mathrm{a} 1,92)$ \\
\hline -EA serios & $34(3.248)$ & $2,94(2,18 \mathrm{a} 3,96)$ \\
\hline -Pérdidas por EA & $23(2.755)$ & \\
\hline Efectos adversos agrupados & & $3,17(2,20 \mathrm{a} 4,58)$ \\
\hline -Del sistema nervioso central & $10(1.521)$ & $1,78(1,43 \mathrm{a} 2,22)$ \\
\hline -Gastrointestinales & $10(1.950)$ & $1,01(0,51 \mathrm{a} 2,0)$ \\
\hline -Muerte & $5(929)$ & \\
\hline
\end{tabular}

EA: efectos adversos

\section{Conclusiones}

Utilizando el sistema GRADE, el estudio calificó a la evidencia que apoya el uso de cannabinoides en el tratamiento del dolor crónico y la espasticidad, como de calidad moderada. Por otro lado los estudios que demostraron beneficios en el tratamiento de las náuseas y los vómitos debido a la quimioterapia, el aumento de peso en pacientes $\mathrm{VIH}$, los trastornos del sueño y el síndrome de
Tourette fueron evaluadas como de baja calidad.

Los cannabinoides se asociaron a un aumento de riesgo de presentar EA a corto plazo.

Fuente de financiamiento/Conflicto de intereses: Oficina Federal de Salud Pública de Suiza en virtud de concesión acuerdo. No hubo conflicto de intereses. 


\section{Comentario}

Los autores de este estudio siguieron rigurosas recomendaciones metodológicas ${ }^{1,2}$ para la realización del mismo y en orden de reducir el riesgo de sesgo de publicación, entre cosas, realizaron una búsqueda altamente sensible. Esto, sin embargo determinó la inclusión de estudios muy heterogéneos, lo que dificultó el análisis en innumerables ocasiones. Además, si bien lograron metaanalizar algunos ensayos, observando los estudios individuales para las cuatro principales indicaciones aquí detalladas, se encontró que sólo el $5 \%$ de los ensayos clínicos presentaba bajo riesgo de sesgo, mientras que el $70 \%$ presentaba alto ries-

\section{go de sesgo.}

Con respecto a los efectos adversos se analizaron los mismos para las dosis más altas de cannabinoides reportadas, no siendo éstas necesariamente las más utilizadas. Una de las desventajas de este estudio es que muchas de las comparaciones agrupadas fueron contra placebo, por lo cual es difícil estimar si hay un efecto adicional con respecto a los tratamientos estándar para los resultados evaluados.

Juliana Camoirano [ Senvicio del Medicina Familiar y Comunitaria del Hospital Italiano de Buenos Aires, juliana.camoirano@ hospitalitaliano.org.ar ]

Camoirano J. El uso medicinal de cannabinoides no demostró un claro beneficio mientras que sí hubo asociación con un riesgo aumentado de presentar efectos adversos. Evid Act Pract Ambul. 2016;19(1):17-18. Ene-Mar. Comentado de: Whiting PF, y col. Cannabinoids for Medical Use: A Systematic Review and Meta-analysis. JAMA. 2015;313(24):2456-73. PMID: 26103030.

\section{Referencia:}

1. Higgins JPT, Green S, eds. Cochrane Handbook for Systematic Reviews of Interventions. Version 5.1.0 (updated March 2011). The Cochrane Collaboration website. Disponible en URL: http://handbook.cochrane.org/.

2. Centre for Reviews and Dissemination. Systematic Reviews: CRD's Guidance for Undertaking Reviews in Health Care (Internet). York, England: University of York; 2009. Disponible en URL: https://www.york.ac.uk/media/crd/Systematic_Reviews.pdf.

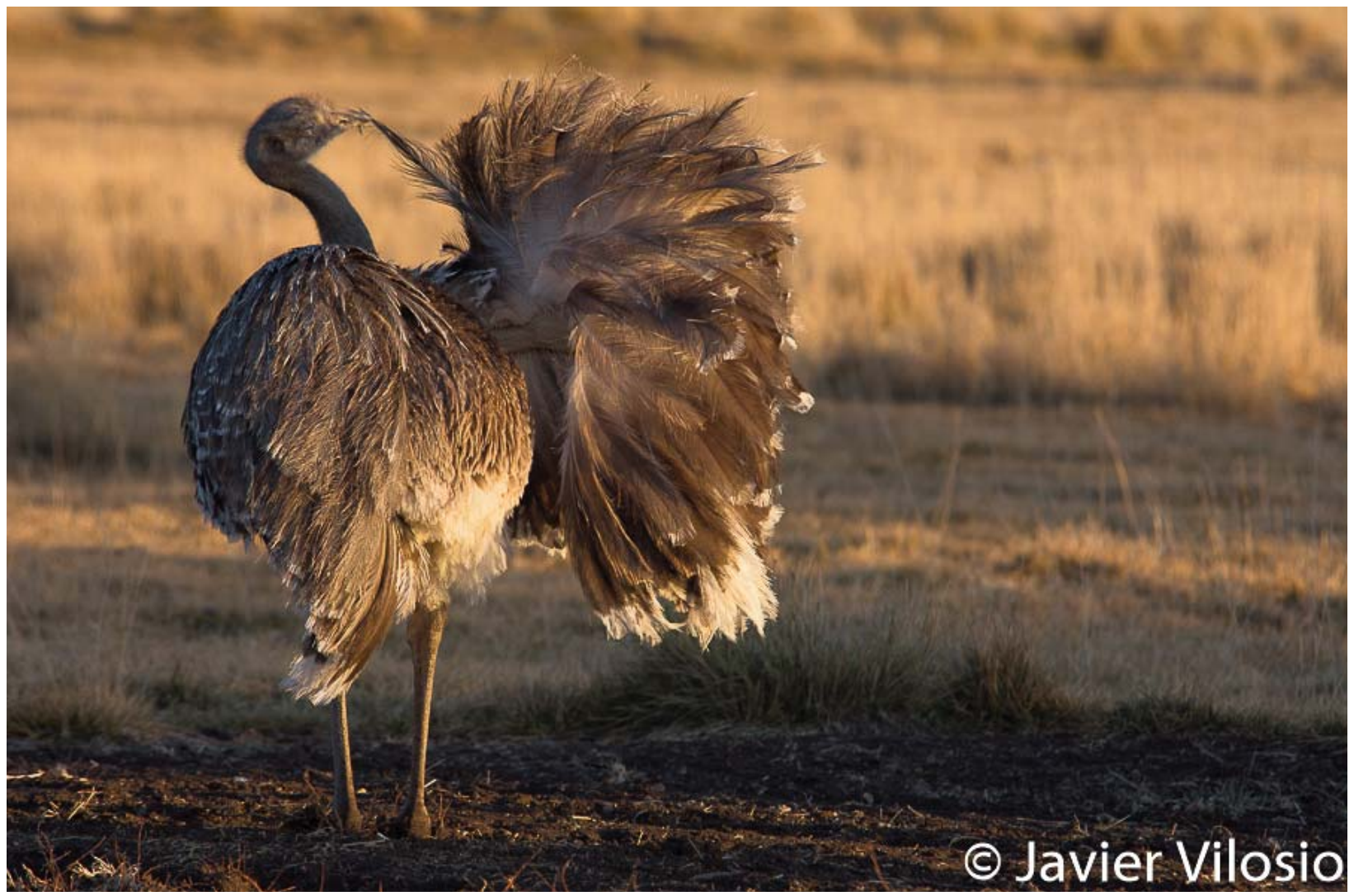

Fotografía: Javier Vilosio 